\title{
Development of Data Acquisition System for Vehicle Power Battery Based on Virtual Instrument
}

\author{
Lijie Chen ${ }^{1, a}$, Yanmin $\mathrm{Xu}^{1,2, \mathrm{~b}}$
}

${ }^{1}$ School of Automobile, Guangdong Mechanical and Electrical College, Guangzhou 510515, China;

${ }^{2}$ School of Mechanical \& Automotive Engineering, South China University of Technology, Guangzhou 510640, China

achenlijie168@163.com, bxu.yanmin@mail.scut.edu.cn

\begin{abstract}
Based on the hardware structure of PCI bus and the software system with hierarchical management and multi-thread technology, the data acquisition system of power battery for multi-channel parallel and high speed data acquisition can be realized. The acquisition system has the characteristics of flexibility, strong compatibility and high reusability of the virtual instrument, which can improve the objectivity and automation of the experiment process.
\end{abstract}

Keywords: Virtual instrument; power battery; data acquisition; electric vehicle.

\section{Introduction}

Virtual instrument (VI) allows users to design their own instrument system according to their own needs, making full use of computer technology to achieve and extend the functionality of the traditional instrument [1]. Virtual instrument is composed of graphical interface software and the computer hardware resources, and is widely used in measurement and control field, helps engineers to solve a variety of problems that the traditional instruments encountered. For example, Chen Lei developed the EPS Central Monitoring System Based on Virtual Instrument [2].

In the process of battery performance test and battery management system inspection, real-time data acquisition system with high precision is needed to acquire the parameters of power battery such as voltage, temperature and current. Real-time monitoring achieves the failures of the power battery system to diagnose faults of system and provide a reliable basis for further analysis of the battery performance and determine the battery management system working normally or not. At present, high precision testing equipment used in power battery testing applications is still relatively deficiency, and the price is expensive.

Lithium ion batteries are still very popular and the most promising technology for the energy storage of the individual mobility of the near future [3]. Researchers of related fields have carried out a series of research on lithium ion battery protection board calibration and test based on virtual instrument, battery internal resistance test etc., making full use of the virtual instrument multi-thread technology, intuitive graphical human-machine interface technology etc.. Compared with the traditional data acquisition system, the system has the advantages of low cost, high sampling precision, easy control, flexible use, and so on.

\section{General Construction}

\subsection{Virtual Instrument for Lithium Ion Power Battery Test.}

The R \& D team of this paper developed a virtual instrument for lithium ion power battery based on PCI bus data acquisition system, which can be used for real-time monitoring and analysis of the parameters of power battery, with the test precision and high sampling speed easy to be guaranteed. The results can be used not only for the experimental equipment of electric vehicles on curriculum construction of colleges and universities, but also for the development of electric vehicle products and personnel re-search to carry out relevant research work. 


\subsection{Key Technical Problems.}

So many parameters of lithium ion battery electrical performance to be test, the traditional manual test method and the multi-level structure testing system of battery manufacturer can't meet the high precision requirements of laboratory testing.

Data acquisition system plays a significant role in modern industrial control and monitoring [4]. Benefited from high speed multi-channel parallel data acquisition and multi thread technology, Data acquisition technology based on PCI bus can be used to develop virtual instrument of electric vehicle battery experiment online data acquisition system, in the case of limited hardware resources. Flexible and customized test plan can be obtained to meet a variety of test requirements of electric vehicle power battery and to provide the basis for the performance analysis of power battery and its management system, solving the problems of low speed samples, high cost, high labor intensity, data analysis ability deficiency in the process of testing. The acquisition system has high flexibility, compatibility and reusability, and the acquisition result has strong objectivity.

The virtual instrument developed for automatic collecting and testing of lithium ion power battery parameters, can be supported by hardware such as programmable power supply, electronic load, host computer and PCI bus data acquisition cards. The power supply and electronic load was controlled through the CAN bus communication protocol. At the same time, real-time acquisition of primary parameters, software filtering technology and mathematical algorithm can be obtained based on high speed data acquisition system of PCI bus. The performance of the cell can be analyzed in the following work according to the test results by comparing with the original battery parameters, so as to know the battery management system is working normally or not.

\subsection{Data Acquisition System Hardware.}

Power battery data acquisition system hardware mainly includes the main controller, PCI bus data acquisition card, signal conditioner, a variety of sensors, CAN bus communication card, etc., as shown in figure 1 . The high precision and high speed parallel data acquisition card based on PCI technology is used to form the signal acquisition layer, and the data is transmitted to the main controller through the PCI bus.

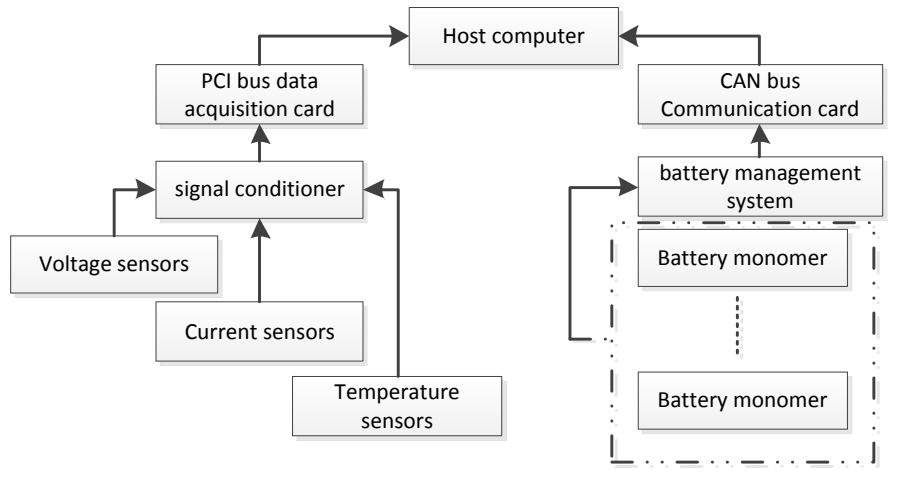

Fig. 1 Power battery data acquisition system hardware

According to the characteristics of lithium ion battery, the voltage range during the charge and discharge process mainly lies between $2.5 \mathrm{~V} \sim 5 \mathrm{~V}$, the signal acquisition and conditioning module can use Advantech PCI-1747U type PCI analog input card for lithium ion power battery voltage acquisition. It has 64 single ended or 32 differential 16 bit high resolution analog input channels, with the sampling rate of $250 \mathrm{kS} / \mathrm{s}$, to provide continuous and uninterrupted streaming data, and high speed sampling for A/D.The working current of the battery is collected by the hall type current sensor, which is matched with the PCI bus analog input acquisition card.

\subsection{Communication with the Battery Management System.}

The parameters of the battery management system is collected by CAN bus output, therefore, data communication between the host computer and the battery management system adopts PCI-1682U type CAN communication, to grasp the battery management system working condition. PCI-1682U communication card is the 2 port CAN bus universal support open CAN protocol PCI communication card, which is widely used in the field of industrial measurement and control. 
Based on the temperature sensor DS18B20 temperature inspection module, the battery temperature signal can be collected, and through the bus interface card and host computer communication, the battery temperature data can be transmitted to the main control unit.

\section{Design of Graphical Interface and Flowchart}

\subsection{Design of Graphical Interface.}

Graphical interface is the main component of human computer interaction of virtual instrument (shown in Figure 2). Tab controller is used in the front panel, which contains of main screen interface, battery parameter monitoring screen interface, battery fault diagnosis screen interface, expert database screen interface, and system composition screen interface.

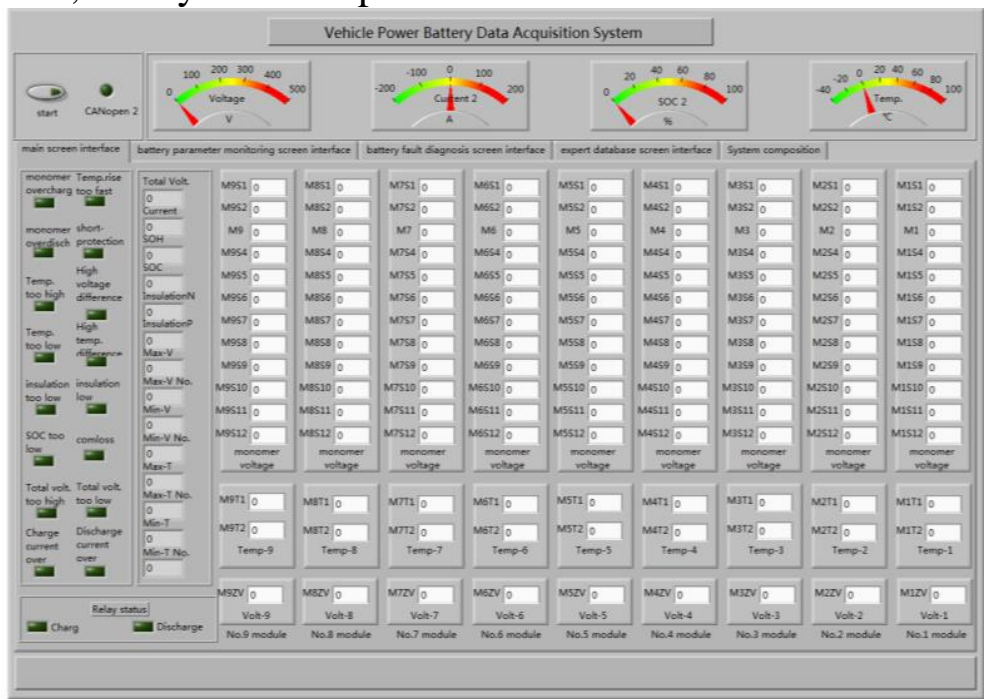

Fig. 2 Main screen interface

\subsection{Flow Chart of Data Acquisition Based on CAN Communication.}

Through CAN communication function of the battery management system, various parameters such as voltage, current, temperature and SOC of the battery can be obtained. Multi-thread technology can be used to achieve data acquisition based on CAN bus, the main thread for the CAN communication data receiving (Figure 3), the secondary thread for CAN bus data analysis (Figure 4).

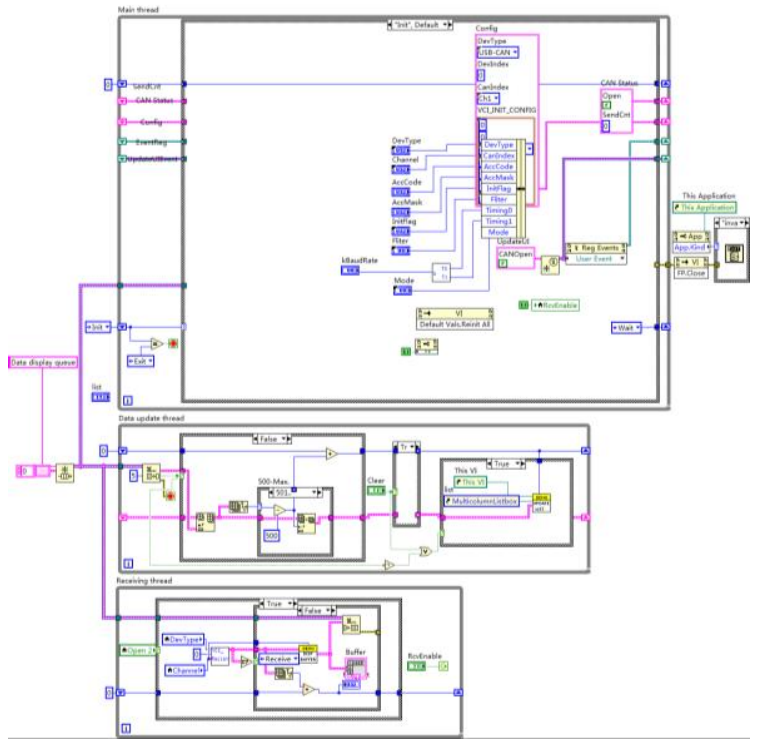

Fig. 3 CAN communication data receiving thread

The former mainly completes the initialization of the CAN communication interface card and the receiving and sending of the CAN bus data. The received data is send to the data analysis thread by the local variables way, for sorting and analysis of data according to CAN communication protocol. 


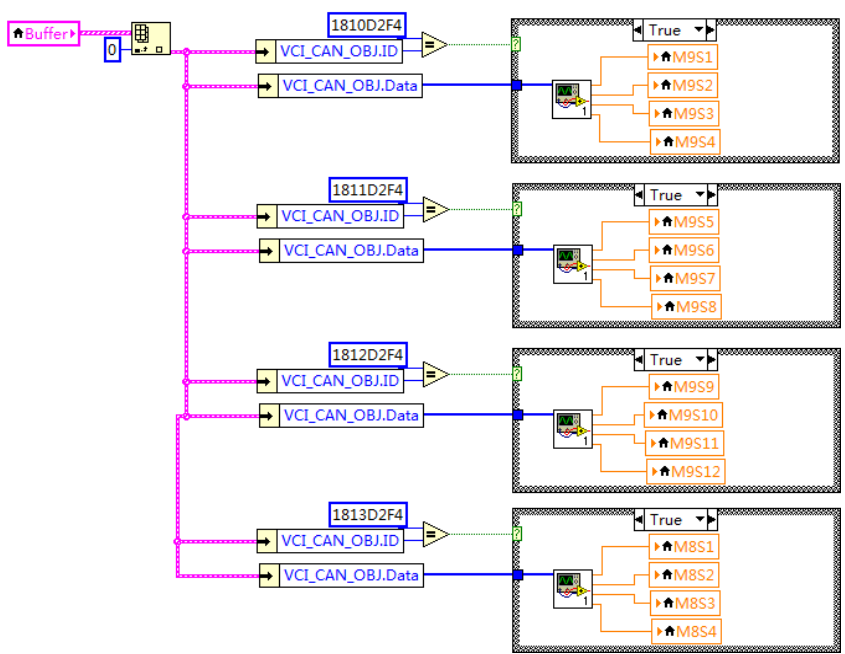

Fig. 4 CAN bus data analysis thread

The parameters of the battery monomer can also be obtained through the PCI data acquisition card. The disadvantage of this method is that only the primary parameters can be obtained, and the secondary parameters need to be calculated.

If the main purpose of the data acquisition is monitoring the fault of single battery, or the abnormity of battery management system, it needs only to compare the parameters obtained by the above two methods. If you want to validate the accuracy of the secondary parameters of a battery management system such as $\mathrm{SOH}$ [5], complex functions should be developed for calculating the secondary parameters from the primary parameters obtained through the PCI data acquisition card. Calculate the secondary parameters in accordance with the algorithm of the battery management system or other more accurate algorithm, and then the computing results can be compared with the output of battery management system itself.

\section{Conclusion}

Based on the data acquisition system of PCI bus, high precision and low cost virtual instrument can be developed, meeting the requirements of high speed data acquisition of power battery parameters in the condition of limited hardware, solving the problems of the conventional single function test instrument. The data acquisition system can be extended by adding the secondary parameters calculation functions. The intuitive and easy human-machine interface built through the graphical programming language, can greatly reduce the testing workload, avoid the wrong operation in the test process, and improve the efficiency of testing.

\section{References}

[1] S. Sumathi, P. Surekha. LabVIEW based Advanced Instrumentation Systems. Springer Berlin Heidelberg, 2007, p1-31.

[2] Lei Chen, Shuang Chen, BaoRu Han. EPS Central Monitoring System Based on Virtual Instrument. In David Jin and Sally Lin (eds.), Advances in Mechanical and Electronic Engineering, Springer Berlin Heidelberg, 2012, p. 183-188.

[3] Hu, X., Sun, F. \& Zou, Y. J. Online model identification of Lithium-ion battery for electric vehicles. Journal of Central South University of Technology. Vol. 18(2011) No.5, p. 1525-1531.

[4] Yinhan Gao, Junjie Gu, Kaiyu Yang, et al. The High-Speed Data Acquisition System Based on LabVIEW and PCI. International Conference, International Conference on Communication and Information Processing. Aveiro, Portugal, March 7-11, 2012, p. 171-178. 
[5] Karsten Mueller, Daniel Tittel , Lars Graube, et al. Optimizing BMS Operating Strategy-Based on Precise SOH Determination of Lithium Ion Battery Cells. In SAE-China and FISITA (eds.), Proceedings of the FISITA 2012 World Automotive Congress: Volume 4: Future Automotive Powertrains (II), Springer Berlin Heidelberg, 2013, p807-819. 